\title{
Mapping of the Insomnia Severity Index and other sleep measures to EuroQol EQ-5D health state utilities
}

\author{
Ning Yan Gu${ }^{1 *}$, Marc F Botteman', Xiang $\mathrm{Ji}^{1}$, Christopher F Bell ${ }^{2}$, John A Carter ${ }^{1}$ and Ben van Hout ${ }^{3,4}$
}

\begin{abstract}
Background: This study sought to map the Insomnia Severity Index (ISI) and symptom variables onto the EQ-5D.

Methods: A cross-sectional survey was conducted among adult US residents with self-reported sleep problems. Respondents provided demographic, comorbidity, and sleep-related information and had completed the ISI and the EQ-5D profile. Respondents were classified into ISI categories indicating no, threshold, moderate, or severe insomnia. Generalized linear models (GLM) were used to map the ISI's 7 items (Model I), summary scores (Model II), clinical categories (Model III), and insomnia symptoms (Model IV), onto the EQ-5D. We used 50\% of the sample for estimation and $50 \%$ for prediction. Prediction accuracy was assessed by mean squared errors (MSEs) and mean absolute errors (MAEs).
\end{abstract}

Results: Mean (standard deviation) sleep duration for respondents $(N=2,842)$ was $7.8(1.9)$ hours, and mean ISI score was 14.1 (4.8). Mean predicted EQ-5D utility was 0.765 (0.08) from Models I-III, which overlapped with observed utilities 0.765 (0.18). Predicted utility using insomnia symptoms was higher $(0.771(0.07))$. Based on Model I, predicted utilities increased linearly with improving ISI (0.493 if ISI = 28 vs. 1.00 if ISI =0, $p<0.01$ ). From Model II, each unit decrease in ISI summary score was associated with a $0.022(p<0.001)$ increase in utility. Predicted utilities were $0.868,0.809,0.722$, and 0.579 , respectively, for the 4 clinical categories, suggesting that lower utility was related to greater insomnia severity. The symptom model (Model IV) indicated a concave sleep-duration function of the EQ-5D; thus, utilities diminished after an optimal amount of sleep. The MSEs/MAEs were substantially lower when predicting EQ-5D $>0.40$, and results were comparable in all models.

Conclusions: Findings suggest that mapping relationships between the EQ-5D and insomnia measures could be established. These relationships may be used to estimate insomnia-related treatment effects on health state utilities.

Keywords: Insomnia, Mapping, Insomnia Severity Index, EQ-5D

\section{Introduction}

Insomnia is a disorder broadly defined by difficulty with sleeping. It may be characterized by 1 ) primary insomnia, without underlying medical cause; 2) secondary insomnia, with presence of an underlying medical cause; 3) acute insomnia, symptoms with a short duration or; 4) chronic insomnia, symptoms with a long duration $[1,2]$. Patients with insomnia commonly complain of

\footnotetext{
* Correspondence: ngu@pharmerit.com

'Pharmerit North America, LLC,4350 East West Highway, Suite 430, Bethesda, MD 20814, USA

Full list of author information is available at the end of the article
}

difficulties initiating/maintaining sleep, early awakening, and non-restorative or poor quality sleep [3].

The prevalence of insomnia in the adult population ranges from $10 \%$ to $30 \%$ [1,4-6]. Insomnia is associated with substantial burden to patient and society. Persistent or prolonged sleeping problems have been associated with worsened health outcomes including reduced productivity or physical/social functioning, increased risk of occupational accidents or major depression/anxiety disorders, poorer health-related quality-of-life (HRQoL) and, increased health care costs [7-13]. Meanwhile, sleep-related conditions have often been under-diagnosed and under-treated [14].

\section{() Biomed Central}


A number of insomnia-related generic and diseasespecific instruments have been used to identify and describe the condition. These instruments include, but are not limited to, the 36-Item Short-Form Health Survey [15], the Leeds Sleep Evaluation Questionnaire [16], the Medical Outcomes Study Sleep Scale 12 [17], the Epworth Sleepiness Scale [18,19], the Functional Outcomes of Sleep Questionnaire [20], the Pittsburgh Sleep Quality Index [21], and the Insomnia Severity Index (ISI) [22]. In addition to these instruments, insomnia symptom variables such as total sleep duration, sleep latency, number of nighttime awakenings, and the affect of prior night's sleep on next-day-sleepiness are predominant indicators of insomnia severity and are routinely collected in clinical studies of insomnia [7].

Among the various instruments used for describing insomnia, the ISI is one of the most commonly used disease-specific measures for self-perceived insomnia severity [23]. The ISI has 7 items describing insomniarelated health impairments concerning 1) difficulty falling asleep; 2) difficulty staying asleep; 3) waking up too early; 4) satisfaction with one's current sleep pattern; 5) self-perceived noticeability of current sleep problems to others with regard to patient's quality-of-life; 6) psychological burdens, and; 7) interference of sleep problems with one's daily functioning. Each item is rated on a 5point Likert scale with scores ranging from 0 to 4 , indicting "none", "mild", "moderate", "severe" and "very severe" sleep problems, respectively. The total ISI score is calculated by summing the scores from the 7 items, and range from a minimum of 0 to a maximum of 28 , with higher scores reflecting more severe sleep problems. In clinical assessments, the ISI total summary score falls into 1 of 4 ISI categories; with scores 0-7, 8$14,15-21$, and 22-28 indicating no clinically significant insomnia, sub-threshold insomnia, moderate insomnia and, clinically severe insomnia, respectively. The psychometric properties of the ISI have been evaluated in earlier studies and have been reported to have sound measurement quality for measuring perceived insomnia severity and the impact of insomnia in different populations $[22,24,25]$.

To quantify the impact of insomnia severity in economic studies such as cost utility analyses (CUA), preference-based measures are required to capture patient preferences for a particular health state [26]. Preferencebased measures can be used to generate health state utilities based on a continuous scale whereby a utility of 1.00 represents "full" health and a utility score of 0.00 corresponds to "death". Such anchored scores are necessary to calculate quality-adjusted life-years (QALYs), a measure of life adjusted for the quality of that life, so that cross comparisons of different health care outcomes are permitted in health economic evaluations [26-28].
Following guidance issued by the National Institute for Health and Clinical Excellence (NICE, 2004) [29] in the United Kingdom, preference-based measures such as the EuroQol EQ-5D [30-32] or the Health Utility Indices [33] have become common means of generating health state utilities.

In particular, the EQ-5D is cognitively simple and takes only a few minutes to complete without imposing excessive response burden. It consists of five items describing health in terms of mobility, self care, usual activities, pain/discomfort, and anxiety/depression. Each item has 3 levels whereby higher levels indicate greater health deficits $(1=$ no problem, $2=$ some problem and 3 = extreme problem). Hence, the EQ-5D descriptive system defines a total of $243\left(3^{5}\right)$ health states. Utility values can be computed from EQ-5D item responses using scoring algorithms [31,34]. Earlier studies have used the EQ-5D in insomnia-related studies for different populations, but mostly for secondary insomnia involving comorbid medical conditions such as depression or cancer $[35,36]$.

CUAs have recently been conducted in the field of insomnia research [7,37-41]. Nonetheless, the evidence regarding the relationship between objective and subjective sleep measures and quantifiable insomnia-related health economic outcomes remains limited. In cases where direct evidence elicited by preference-based measures is not available, establishing a mapping relationship between descriptive clinical measures on insomnia and quantitative effects of insomnia on HRQoL can be useful.

The purpose of the present study was therefore to establish such a mapping relationship between insomnia-related measures and the EQ-5D. We aimed to estimate the associations between the EQ-5D health state utilities and insomnia severity measures by mapping the ISI and/or predominant indicators of insomnia onto the EQ-5D.

\section{Methods}

\section{Survey}

The analysis was based on a cross-sectional internet survey of approximately 3,000 US residents with signs and symptoms suggestive of chronic insomnia. This was an observational study designed to explore the relationship between subject-reported sleep measurements and outcomes (i.e. quality of life, functionality, and impact of sleep) in the US community. The survey was fielded by Harris Interactive which maintains a proprietary webenabled panel of research subjects in the US who have agreed to participate in ongoing survey research.

Prior to the screening of any potential subjects, a central Institutional Review Board (IRB) approved the protocol (GHO-2008-008, 1/5/09), informed consent form, 
survey instruments, and all other subject information and/or recruitment materials. To recruit participants, e-mail invitations were sent to approximately 90,000 panel members representative of the general public. It was estimated that approximately $20 \%$ of the panel members in the specified subset would respond to the e-mail invitation. Of those, a $60 \%$ qualification rate was assumed among those insomnia-diagnosed patients. Overall, approximately 3,000 subjects were expected to enroll and complete the study. The study duration was estimated to be roughly 8 weeks, which included time for subject recruitment and completion of the questionnaire.

\section{Data}

Subjects completed a questionnaire that collected information on demographics, comorbidities, and previousnight sleep symptoms. Subjects also provided responses to the ISI and the EQ-5D. Subjects with complete responses on the EQ-5D and the ISI questionnaire were included in the study if they a) were at least 18 years of age; b) provided informed consent to participate in the survey; c) were at least moderately bothered by their sleep problems; d) had reported problems with (i) falling asleep at the beginning of the night (ii) staying asleep throughout the night or (iii) not feeling refreshed upon waking following what was expected to be an adequate night's sleep for at least 3 times per week, or (iv) at least 2 of the problems listed above at least once per week.

Subjects were excluded from the study if they 1) were employed in full-time or part-time jobs that involved night shifts or day-night rotating shifts; 2) had children under 1 year old; or 3) reported a physician-diagnosis of competing symptoms of sleep such as obstructive sleep apnea, narcolepsy, periodic limb movement disorder, or restless leg syndrome.

\section{Models and Variables}

A series of generalized linear models (GLM) was used for the present analysis. Based on the distribution of the variables, we indentified a gamma family distribution and a log link using the Modified Park tests for model specifications $[42,43]$. The dependent variable was the EQ-5D utilities computed based on the responses to the 5 items using a US algorithm [34]. While the gamma family was selected to account for the skewed dependent variable distribution, to respect its distribution for real values on a positive space (from 0 to $\infty$ ) [43], the modeled dependent variable was constructed as the disutility values of the EQ-5D (= 1-utility) computed using the following equation:

$$
\text { Utility }=1-\text { Disutility }=1-\exp \left(\alpha+\sum X i \beta\right)
$$

Four GLM functional forms were used (Table 1). For Models I-III the predictors for the EQ-5D disutility values were the 7 ISI items, a continuous (0-28) ISI summary score, and the 4 ISI clinical categories, respectively. For Model IV, we used sleep symptom variables identified from the existing literature on insomnia [7,44-46], namely, previous night's sleep duration, sleep quality, sleep latency, next-day-sleepiness as an effect of prior night's sleep, and the number of wakeup times during the night. Predictors in Model IV were supplemented with patient characteristics such as age, gender, and the presence/absence of comorbidities.

The comorbidity predictor was constructed as a binary variable representing the presence $(=1)$ or absence $(=0)$ of any of the 17 chronic non-insomnia-competing conditions reported by the respondents based on prior physician diagnoses. The chronic conditions included: anxiety disorder, arthritis, bipolar disorder, cancer, cardiovascular condition, chronic fatigue syndrome, chronic pain, depression, diabetes, drug/alcohol abuse, fibromyalgia, HIV/AIDS, insomnia, irritable bowel syndrome, neuropathic pain, respiratory condition, and schizophrenia.

The decision to use a single binary(yes/no) comorbidity presence indicator rather than one variable per condition or counting the sum of the total number of conditions was made, primarily, to impose a minimal burden on future data collection. Specifically, it should be emphasized that the objective of this study was not to predict utility levels using a large number of clinical and demographic variables. Rather, we sought to construct a simple-if not generic-tool that would allow researchers to predict utility in a community-based population of individuals exhibiting insomnia symptoms using as few variables as possible. Ultimately, we hope that the algorithm generated in this process can be used by researchers who either could not collect utilities in previous research or, for other reasons, will not be able to do so in the future. Hence, the focus of this analysis was on external rather than internal validity. The approach selected herein with regard to comorbidity was consistent with the broader sleep-research literature which emphasizes insomnia without comorbidities (i.e., primary insomnia) from insomnia with comorbidities (i. e., secondary insomnia).

For the sleep duration variable used in the Model IV, based on preliminary analysis of the predictors, observed EQ-5D health state utility was found to be optimal when the amount of sleep was approximately 7-9 hours (mean [standard deviation, SD] sleep duration $=7.8$ [1.9] hours). EQ-5D utility decreased when one slept more/ less than that optimal amount or extreme hours, which gave a concave sleep duration function of EQ-5D utility/ 


\section{Model I: ISI 7 items}

disutility. Thus, a squared term of the sleep duration variable was included in the model.

Moreover, the sleep quality-rating variable ranged from 0 , indicating poor sleep quality to 10 , indicating excellent sleep quality. The next-day-sleepiness item also used a rating ranging from 0 suggesting not feeling sleepy due to prior night's sleep pattern to 10 for feeling extremely sleepy. Sleep latency was captured using total minutes of delay to sleep and the total number of wake up times during the night ranged from minimum of 1 time to a maximum of 5 times. We treated these predictors as continuous for simplicity.

\section{Analyses}

We used $50 \%$ of the sample to generate the mapping function (i.e., estimation sample) and, the other $50 \%$ to validate the model performance (i.e., validation sample). Samples were randomly split for each process. Predictions in the validation process were made based on parameters estimated from estimation sample. The validation process was repeated 30 times to ensure we obtained a sufficient number of predictions to account for variability. Average values from the 30 validations were calculated for each of the 4 models.
To determine the predictive precision of the models, we computed model mean square error (MSE) and mean absolute error (MAE). The MSE was given by: $M S E=\frac{\sum(Y-\hat{Y})^{2}}{N-K}$ where $\mathrm{Y}=$ observed EQ-5D values, $\hat{Y}=$ predicted EQ $-5 \mathrm{D}$ values, $\mathrm{N}=$ total or subgroup sample sizes and, $K=$ degrees of freedom. Hence, the MSEs were computed by adjusting the number of independent variables included in the model. Thus, a perfect prediction would be indicated by a zero MSE and, smaller MSEs indicated lower prediction errors. Since MSEs penalized larger errors by using the squared term of errors, we also computed the MAE. The MAE was given by: $M A E=\frac{\sum|Y-\hat{Y}|}{N}$, again, $\mathrm{Y}=$ observed EQ-5D values, $\hat{Y}=$ predicted $E Q-5 D$ values $\mathrm{N}=$ total or subgroup sample sizes, The MAEs provided error statistics that did not give greater weight to larger errors.

The overall model performance was also assessed by examining the distributional qualities of the predicted EQ-5D scores compared with the observed ones with regard to mean, median, $\mathrm{min} / \mathrm{max}$, and range. SAS 9.2 was used for data preparation, STATA11 was used for 
regression and statistical tests, and Microsoft Excel was used for prediction error computations.

\section{Results}

Of the 3,034 survey participants, 2,842 (93.67\%) met all inclusion/exclusion criteria and were included in the present analyses. Table 2 reports respondents' sociodemographic characteristics. The mean $( \pm$ SD) age of the total sample was 42.9 ( \pm 15.7 ) years. Nearly two thirds of respondents were female and $83.7 \%$ were Caucasian. The mean $( \pm \mathrm{SD})$ observed EQ-5D utility score was $0.765( \pm 0.20)$ and was lowest for individuals aged 50 to $59(0.749[ \pm 0.20])$. On average, respondents reported $7.8( \pm 1.9)$ hours of sleep during the previous night. The mean $( \pm$ SD) ISI summary score was 14.1 ( \pm 4.8). According to the ISI clinical classification, $6.19 \%$, $52.08 \%, 33.57 \%$, and $8.16 \%$ of respondents were categorized as having no clinically significant insomnia, subthreshold insomnia, moderately severe insomnia, and severe insomnia, respectively. Individuals aged 40 to 59 reported the least mean $( \pm S D)$ amount of sleep duration (7.5 $[ \pm 1.8])$ and higher scores on ISI items, suggesting greater sleep problems. In addition, compared with male respondents, females reported longer mean sleep durations (7.9 vs. 7.5 hours) and higher mean EQ5D scores (0.766 vs. 0.762$)$ despite higher ISI summary scores (14.3 vs. 13.7). In this particular sample $62.4 \%$ of respondents reported $\geq 1$ comorbid medical conditions and, for those who reported $\geq 1$ comorbid conditions, mean EQ-5D scores were lower compared with those who reported no comorbid conditions ( 0.723 vs. 0.835 ). ISI summary scores decreased (i.e., insomnia improved) as sleep quality improved, and increased (i.e., insomnia worsened) with rating increases of next-day-sleepiness, sleep latency, and number of wake-up times. Therefore, a longer time to fall asleep and a greater number of wake-up times during a night were associated with higher ISI scores and lower the EQ-5D utility values.

Table 3 exhibits the model regression estimates using the different set of predictors on EQ-5D disutility. For Model I, except for ISI item 4 (i.e., degree of satisfaction with sleep pattern) and item 7 (extent of interference with daily functioning due to sleep pattern), ISI items showed significant associations with EQ-5D scores ( $\mathrm{p}<$ 0.05). Higher levels of ISI items were associated with greater health deficit (greater disutility values). ISI summary scores in Model II and clinical classification scores in Model III also showed significant associations with EQ-5D disutility values $(\mathrm{p}<0.001)$. In Model IV, except for age and gender, all other predictors were able to provide substantial explanations of EQ-5D scores ( $\mathrm{p}<$ $0.05)$. The signs of model coefficients were as expected.

Table 4 compares the mean, median, $\mathrm{min} / \mathrm{max}$, and range of observed and predicted EQ-5D values for both estimation and validation sample estimates. EQ-5D utility values were computed using equation (1). The observed EQ-5D mean $( \pm$ SD) utility value was $0.765( \pm$ 0.18 ), with a median value of 0.800 and a range of [-0.040-1.000]. Mean predicted scores were identical with those observed for three ISI models (Models I-III). The predicted mean was slightly higher for Model IV, when insomnia symptoms were used for prediction (0.771 $( \pm 0.07))$, compared with Models I-III using ISI $(0.765( \pm 0.08))$. Similar results were found in validation sample estimates.

Table 5 shows the model prediction errors. The MSEs and MAEs were lower for EQ-5D utilities greater than 0.40 . This suggested that predictions were more robust for higher health state utilities (i.e., $>0.4$ ), which were reported by $94.02 \%$ of the respondents in the sample. At the same time, relatively poorer predictions at tail end of utilities have been reported as a common problem associated with mapping [47]. Owing to the very small sample size $(0.63 \%)$ of respondents who had observed utilities < 0.2, MSEs for EQ-5D utilities less than 0.2 could not be computed in Models I and IV. Similar results were found using the validation samples.

Figure 1 depicts observed and predicted EQ-5D utility values for Models I-IV along the ISI scores (ranging from 0 to 28), which showed negative correlations between the two instruments. The predicted EQ-5D utility values followed more closely with observed ones for mid-range scores, especially for Models I-III. For Model IV, EQ-5D utility values were under-predicted for ISI scores less than 14 (no insomnia or threshold insomnia) and over-predicted for ISI scores greater than 14 (moderate or severe insomnia). Nevertheless, the association between greater insomnia symptom severity and lower health utilities was observed in all models.

Figure 2 shows the observed and predicted EQ-5D values along sleep duration using half-hour intervals. Again, predicted and observed EQ-5D utilities followed each other closely along sleep durations except for the higher and lower ends of sleep hours (i.e., extreme hours). The concave function of sleep duration of the EQ-5D utility was preserved, suggesting that health state utilities increase at a decreasing rate along sleep durations.

\section{Discussion}

The extent of association between a disease-specific instrument (i.e., ISI) and a generic preference-based instrument (i.e., EQ-5D) is affected by the amount of correspondence between the two measures with regard to the underlying HRQoL [48]. Findings from this analysis suggest there was sufficient overlapping of underlying HRQoL between insomnia measures and EQ-5D health state utilities. While the ISI is commonly used in clinical 
Table 2 Sample Characteristics

\begin{tabular}{|c|c|c|c|c|c|c|}
\hline & Number & $\%$ & Mean Age (SD) (Years) & $\begin{array}{c}\text { Mean (SD) } \\
\text { EQ-5D }\end{array}$ & Mean Duration (SD) (Hours) & $\begin{array}{l}\text { Mean ISI } \\
\text { (SD) }\end{array}$ \\
\hline Whole sample & 2,842 & 100 & $42.9(15.7)$ & $0.765(0.2)$ & $7.8(1.9)$ & $14.1(4.8)$ \\
\hline \multicolumn{7}{|l|}{ Age group } \\
\hline $18-29$ & 743 & 26.1 & $24.2(3.8)$ & $0.784(0.2)$ & $8.0(1.9)$ & $13.4(4.6)$ \\
\hline $30-39$ & 569 & 20.0 & $34.7(2.9)$ & $0.754(0.2)$ & $7.7(1.9)$ & $15.0(5.0)$ \\
\hline $40-49$ & 624 & 22.0 & $45.0(2.8)$ & $0.754(0.2)$ & $7.5(1.8)$ & $15.0(4.7)$ \\
\hline $50-59$ & 405 & 14.3 & $54.9(2.8)$ & $0.749(0.2)$ & $7.5(1.8)$ & $14.2(4.7)$ \\
\hline $60-69$ & 351 & 12.4 & $64.2(2.9)$ & $0.770(0.2)$ & $7.9(1.9)$ & $12.9(4.5)$ \\
\hline$\geq 70$ & 150 & 5.30 & $74.8(4.6)$ & $0.786(0.1)$ & $8.1(1.6)$ & $12.3(4.7)$ \\
\hline \multicolumn{7}{|l|}{ Gender } \\
\hline Female & 1,834 & 64.50 & $42.0(15.9)$ & $0.766(0.2)$ & $7.9(1.9)$ & $14.3(4.8)$ \\
\hline Male & 1,008 & 35.50 & $44.5(15.2)$ & $0.762(0.2)$ & $7.5(1.8)$ & $13.7(4.7)$ \\
\hline \multicolumn{7}{|l|}{ Race } \\
\hline White & 2,318 & 83.7 & $44.0(15.7)$ & $0.768(0.2)$ & $7.8(1.8)$ & $13.9(4.7)$ \\
\hline Black & 292 & 10.5 & $38.1(14.7)$ & $0.755(0.2)$ & $7.4(2.1)$ & $14.7(5.1)$ \\
\hline Asian & 44 & 1.60 & $26.7(10.4)$ & $0.805(0.1)$ & $7.4(2.1)$ & $13.7(3.7)$ \\
\hline Other & 116 & 4.20 & $38.3(13.7)$ & $0.719(0.2)$ & $7.5(2.1)$ & $15.7(5.3)$ \\
\hline \multicolumn{7}{|l|}{ Comorbidity } \\
\hline No & 1068 & 37.60 & $38.2(14.4)$ & $0.835(0.1)$ & $7.7(1.8)$ & $12.7(4.3)$ \\
\hline Yes & 1774 & 62.40 & $45.7(15.8)$ & $0.723(0.2)$ & $7.8(1.9)$ & $14.9(4.9)$ \\
\hline \multicolumn{7}{|l|}{ Sleep quality } \\
\hline 0 & 146 & 5.14 & $43.6(13.4)$ & $0.614(0.2)$ & $6.6(2.5)$ & $20.5(5.1)$ \\
\hline 1 & 94 & 3.31 & $43.7(15.7)$ & $0.697(0.2)$ & $7.2(2.3)$ & $18.3(4.8)$ \\
\hline 2 & 250 & 8.81 & $43.9(14.2)$ & $0.712(0.2)$ & $7.4(2.2)$ & $17.1(4.6)$ \\
\hline 3 & 457 & 16.10 & $44.0(15.0)$ & $0.755(0.2)$ & $7.7(1.9)$ & $15.5(4.1)$ \\
\hline 4 & 457 & 16.10 & $41.6(14.8)$ & $0.763(0.2)$ & $7.9(1.8)$ & $13.9(4.0)$ \\
\hline 5 & 559 & 19.70 & $43.3(15.7)$ & $0.795(0.1)$ & $7.8(1.6)$ & $12.9(3.9)$ \\
\hline 6 & 411 & 14.48 & 40.9 (16.8) & $0.800(0.1)$ & $8.0(1.6)$ & $12.2(3.7)$ \\
\hline 7 & 291 & 10.25 & $42.7(17.0)$ & $0.804(0.2)$ & $8.0(1.7)$ & $11.6(4.1)$ \\
\hline 8 & 108 & 3.81 & $43.8(17.8)$ & $0.781(0.2)$ & $8.4(1.8)$ & $10.9(4.0)$ \\
\hline 9 & 35 & 1.23 & $40.5(18.3)$ & $0.858(0.2)$ & $8.5(1.2)$ & $11.7(5.2)$ \\
\hline 10 & 30 & 1.06 & 48.3 (17.6) & $0.727(0.3)$ & $8.0(2.2)$ & $10.5(5.7)$ \\
\hline \multicolumn{7}{|c|}{ Next day sleepiness } \\
\hline 0 & 162 & 5.71 & $54.0(15.8)$ & $0.764(0.2)$ & $7.8(2.0)$ & $12.4(5.4)$ \\
\hline 1 & 100 & 3.52 & $49.3(16.6)$ & $0.805(0.2)$ & $8.0(2.0)$ & $12.2(4.5)$ \\
\hline 2 & 157 & 5.53 & $45.0(16.9)$ & $0.792(0.2)$ & $7.9(1.8)$ & $12.5(4.0)$ \\
\hline 3 & 213 & 7.51 & $46.0(16.0)$ & $0.788(0.2)$ & $7.8(1.7)$ & $12.4(4.1)$ \\
\hline 4 & 236 & 8.32 & $44.6(16.1)$ & $0.776(0.2)$ & $7.8(1.8)$ & $13.0(4.1)$ \\
\hline 5 & 373 & 13.15 & $44.4(15.6)$ & $0.781(0.2)$ & $7.9(1.8)$ & $13.5(4.3)$ \\
\hline 6 & 436 & 15.37 & $40.9(15.4)$ & $0.783(0.2)$ & $7.8(1.9)$ & $13.5(4.2)$ \\
\hline 7 & 520 & 18.33 & $40.6(14.2)$ & $0.758(0.2)$ & $7.8(1.8)$ & $14.7(4.6)$ \\
\hline 8 & 364 & 12.83 & $38.4(14.3)$ & $0.747(0.2)$ & $7.7(1.9)$ & $15.6(4.8)$ \\
\hline 9 & 135 & 4.76 & $40.5(13.7)$ & $0.683(0.2)$ & $7.5(2.1)$ & $16.2(5.3)$ \\
\hline 10 & 141 & 4.97 & $39.2(14.0)$ & $0.710(0.2)$ & $7.2(2.2)$ & $18.1(5.5)$ \\
\hline \multicolumn{7}{|c|}{ Latency (minutes) } \\
\hline$<15$ & 443 & 17.39 & $44.2(15.2)$ & $0.808(0.1)$ & $7.7(1.7)$ & $11.9(4.3)$ \\
\hline $15-30$ & 468 & 18.37 & $44.7(16.5)$ & $0.799(0.2)$ & $7.8(1.7)$ & $12.3(4.1)$ \\
\hline
\end{tabular}


Table 2 Sample Characteristics (Continued)

\begin{tabular}{|c|c|c|c|c|c|c|}
\hline $30-45$ & 449 & 17.62 & $42.9(16.0)$ & $0.791(0.1)$ & $7.8(1.7)$ & $13.5(4.3)$ \\
\hline $45-60$ & 205 & 8.05 & $41.8(15.0)$ & $0.773(0.2)$ & $7.6(1.7)$ & $13.9(4.3)$ \\
\hline $60-90$ & 404 & 15.86 & $42.3(15.1)$ & $0.752(0.2)$ & $7.8(1.8)$ & $14.6(4.4)$ \\
\hline$>=90$ & 579 & 22.72 & 40.5 (15.9) & $0.729(0.2)$ & $8.0(2.1)$ & $15.9(4.7)$ \\
\hline \multicolumn{7}{|c|}{ Wake up times } \\
\hline 1 & 375 & 18.20 & $43.6(15.4)$ & $0.802(0.2)$ & $7.5(1.7)$ & $12.8(4.3)$ \\
\hline 2 & 732 & 35.6 & $44.5(16.1)$ & $0.780(0.2)$ & $7.7(1.7)$ & $13.6(4.5)$ \\
\hline 3 & 588 & 28.6 & $44.1(15.6)$ & $0.759(0.2)$ & $8.0(1.8)$ & $14.3(4.4)$ \\
\hline 4 & 184 & 8.90 & 44.9 (15.2) & $0.743(0.2)$ & $8.3(1.8)$ & $15.6(4.7)$ \\
\hline 5 & 179 & 8.70 & $42.6(13.0)$ & $0.715(0.2)$ & $8.4(1.8)$ & $16.2(4.6)$ \\
\hline
\end{tabular}

trials and was specifically designed to describe and evaluate insomnia severity, it does not provide the preference-based measures necessary for health economic evaluations quantifying the impact of insomnia on patients' health state utilities. To the best of our knowledge, the present study is the first to map an insomniaspecific instrument and/or sleep variables to EQ-5D utility values. Hence, the cross-walk conducted herein establishes a preliminary mapping relationship and link between these measures.

Findings from this analysis were consistent with earlier studies wherein poorer sleep outcomes (e.g., sleep quality, next-day-sleepiness, and extreme sleep hours) were significantly associated with declining HRQoL $[8,12,49]$. Such associations were observed in the present analysis, especially for individuals with greater sleep difficulties (Figure 1) or extreme sleep durations (Figure 2).

With respect to sleep duration for example, a recent study by Faubel et al. (2009) reported that for older men and women, extreme sleep durations $(\leq 5$ hours or $\geq 10$ hours) were indicators of worse HRQoL, compared with those who sleep 7-8 hours per night [8]. Studies of different populations have also reported this same effect: that short-duration sleep [50] or long-duration sleep [51] or both [52], were associated with worse HRQoL. This association was noted in our model predictions by taking other factors into consideration (Model IV).

Quantifying the effect of insomnia on HRQoL is essential for targeting effective treatments, and comparing treatment cohorts in clinical practice. The mapping relationship established between the ISI and the EQ-5D in this analysis provides a necessary cross-walk when a preference-based measure such as EQ-5D was not administered. For example, using a hypothetical scenario where a 36-year old female had ISI item scores of $0,3,1,4,3,3$, and 2 , which would result in an ISI summary score of 16 and a ISI class of 3 . Further, she also reported 8.58 hours of sleep, $\geq 1$ comorbidity, 1 on overall sleep quality, 5 minutes of sleep onset latency during previous night, 8 on next day sleepiness, and 5 wake-up times. Using the algorithms provided (Table 1 equation (1)), her estimated EQ-5D utility values would be $0.772,0.748,0.723$, and 0.690 using Models I-IV, respectively (see Table 6 for sample computations).

For this particular scenario, compared with Model I, the estimated utilities using Models II and III were decreased by approximately $3 \%$ and $6 \%$, respectively ( 0.772 vs. 0.748 and 0.723 ). When Model IV was used, the estimated utility was lower by $10 \%$ ( 0.772 vs. 0.690$)$. These discrepancies were associated with different model specifications as each model incorporated different assumptions. In Model I, the predictions based on 7 distinctive item scores were able to capture more precise information for describing the EQ-5D and different items categories were assumed to have their own weight on the EQ-5D scores. Conversely, in Model II a continuous summary score ranging from 0 to 28 was used. This assumed that each unit increment/decrement in total ISI score would give the same impact on the EQ5D regardless of which item. The case is similar for Model III, where the clinical categories were constructed based on the summary scores. One way to conceptualize this is to understand that Models II and III penalized the estimated utilities by placing more weight on the items that had more impact on the EQ-5D. Model IV is a different model specification aimed to estimate the same outcome. It captured more disease-specific information in explanatory variables, and therefore the estimated EQ-5D utilities were penalized the most. Alternatively, these discrepancies could be lowered by using a sample scenario with all best levels of ISI items where no problems were reported on all items, i.e., score $=0$ on all items. Using the same computation, the estimated utilities for Models I-III would be approximately $0.914,0.905$ and 0.868 , respectively, resulting $1 \%$ and 5\% differences in estimated utilities using Models II and III, respectively compared with Model I. Given these findings, Model I should be used when data for all ISI items are available. 
Table 3 Model Estimations

\begin{tabular}{|c|c|c|c|c|}
\hline & Model I & Model II & Model III & Model IV \\
\hline Intercept & $-2.45614(0.27103) * * * *$ & $-2.34909(0.0493) * * * *$ & $-2.0259(0.0733) * * * *$ & $-0.99338(0.17896) * * * *$ \\
\hline \multicolumn{5}{|l|}{$|S| \_1$} \\
\hline 1 & $0.04681(0.05083)$ & - & - & - \\
\hline 2 & $0.14743(0.04637) * * *$ & - & - & - \\
\hline 3 & $0.23814(0.05315)^{* * * *}$ & - & - & - \\
\hline 4 & $0.20228(0.08007) * *$ & - & - & - \\
\hline \multicolumn{5}{|l|}{$|S| \_2$} \\
\hline 1 & $-0.00487(0.06257)$ & - & - & - \\
\hline 2 & $0.10069(0.05168) *$ & - & - & - \\
\hline 3 & $0.1438(0.0581) * *$ & - & - & - \\
\hline 4 & $0.22504(0.08002) * * *$ & - & - & - \\
\hline \multicolumn{5}{|l|}{$\mid \overline{|S|} 3$} \\
\hline 1 & $-0.04075(0.04682)$ & - & - & - \\
\hline 2 & $0.00373(0.04365)$ & - & - & - \\
\hline 3 & $0.13501(0.05178) * * *$ & - & - & - \\
\hline 4 & $0.15058(0.06278) * *$ & - & - & - \\
\hline \multicolumn{5}{|l|}{$|S| \_4$} \\
\hline 1 & $0.30681(0.28409)$ & - & - & - \\
\hline 2 & $0.23039(0.26934)$ & - & - & - \\
\hline 3 & $0.14912(0.26789)$ & - & - & - \\
\hline 4 & $0.13552(0.26478)$ & - & - & - \\
\hline \multicolumn{5}{|l|}{$|S| \_5$} \\
\hline 1 & $0.18721(0.12667)$ & - & - & - \\
\hline 2 & $0.3242(0.12139) * * *$ & - & - & - \\
\hline 3 & $0.41963(0.12265)^{* * *}$ & - & - & - \\
\hline 4 & $0.58239(0.12811) * * * *$ & - & - & - \\
\hline \multicolumn{5}{|l|}{$\overline{|S| \_6}$} \\
\hline 1 & $0.13325(0.0471) * * *$ & - & - & - \\
\hline 2 & $0.16124(0.04784) * * *$ & - & - & - \\
\hline 3 & $0.24329(0.05675)^{* * * *}$ & - & - & - \\
\hline 4 & $0.38139(0.06977)^{* * * *}$ & - & - & - \\
\hline \multicolumn{5}{|l|}{$|S| \_7$} \\
\hline 1 & $0.04661(0.07847)$ & - & - & - \\
\hline 2 & $0.0766(0.07898)$ & - & - & - \\
\hline 3 & $0.11301(0.0838)$ & - & - & - \\
\hline 4 & $0.16077(0.09968)$ & - & - & - \\
\hline ISI summary & - & $0.06077(0.00307)^{* * * *}$ & - & - \\
\hline \multicolumn{5}{|l|}{ ISI class } \\
\hline 2 & - & - & $0.36953(0.07558) * * * *$ & - \\
\hline 3 & - & - & $0.74122(0.07544) * * * *$ & - \\
\hline 4 & - & - & $1.15809(0.08459) * * * *$ & - \\
\hline Age & - & - & - & $-0.00079(0.00103)$ \\
\hline Female & - & - & - & $-0.03204(0.02736)$ \\
\hline Duration & - & - & - & $-0.22818(0.04158) * * * *$ \\
\hline Duration $^{2}$ & - & - & - & $0.0133(0.00265) * * * *$ \\
\hline Comorbidity & - & - & - & $0.42901(0.02861) * * * *$ \\
\hline Sleep quality & - & - & - & $-0.0314(0.00953)^{* * *}$ \\
\hline
\end{tabular}


Table 3 Model Estimations (Continued)

\begin{tabular}{lcccc}
\hline Latency & - & - & - & $0.00112(0.00026)^{* * * *}$ \\
\hline Sleepiness & - & - & - & $0.02498(0.00754)^{* * *}$ \\
\hline Wake times & - & - & - & $0.05213(0.01348)^{* * * *}$ \\
\hline Log-likelihood & 1378.765 & 1405.107 & 1392.894 & 1018.948 \\
\hline
\end{tabular}

For model specifications please refer to Table 1

ISI class_1 = No clinically significant insomnia; ISI class_2 = Subthreshold insomnia; ISI class_3 = Moderate insomnia; ISI class_4 = Se were insomnia

${ }^{*} \mathrm{p}<0.10$; ${ }^{* *} \mathrm{p}<0.05$; ${ }^{* *} \mathrm{p}<0.01$; **** $\mathrm{p}<0.001$

Based on the findings, marginal changes on treatment effects on insomnia could also be estimated. If the same respondent had improved her sleep pattern somehow and decreased her ISI score to 14 (from 16), then by using Model II, her predicted EQ-5D utility score would be $0.776(=1-\exp (-2.34909+0.06077 * 14)$ instead of 0.748 , corresponding to a difference of approximately 0.0289 unit of utility. On the other hand, holding other variables constant and assuming changes to ISI item 5 (e.g., if she thought her sleep problem was noticed "a little by others" (level 1)); then using the Model I algorithm, her health utility would be 0.761 (= 1-exp $(-2.45614+0.04681+0.14380-0.04075+0.13552+0.41963$ $+0.24329+0.0766)$ ), instead of 0.772 . Similar magnitudes of health disutility gains and losses could be estimated if changes had taken place on other ISI items. From a health policy standpoint, such quantification is useful for assessing the effect of treatment on health utility values. Thus, the findings provided herein make it possible to estimate EQ-5D utility values when direct evidence is absent from the primary research, but when ISI or insomnia-related symptoms data are available.

It is important to note the limitations of this study. First, while significant associations were noted between insomnia measures and EQ-5D utilities in the current study, the mapping technique implicitly assumes that the EQ-5D covers all important aspects of the latent health construct that the ISI is intended to measure. Hence, the strength of the mapping function is underpinned by the degree of overlap between the two measures. While all model estimates rendered very close approximations of the EQ-5D observed scores, the regression-based transformation presented herein is selfcontained in that disease-specific scores are permitted to be transformed to a generic utility measure without referencing additional data and aimed for repeated uses on secondary data analysis from other clinical trials [48]. Therefore, the external validity of the study should be verified using other data sets.

Second, since different models control for different predictors, each entails a different magnitude of effects. At the group level, predicted mean utility values closely estimated observed values. At the individual level, algorithms presented in this study may include some discrepancies from one model to another, as was indicated in the above example. Therefore, our algorithms may more accurately predict individual EQ-5D values for a group level evaluation for CUA studies in making group level comparisons.

Third, regression-based mapping from one measure to another inevitably results in floor and ceiling effects for predicted values [48]. We noted higher MSEs in all models for lower EQ-5D utility values $(E Q-5 D<0.4)$ Thus, our algorithms were less reliable for respondents with EQ-5D scores $<0.4$.

Fourth, QALYs implicitly involve the concept of survival, but we estimated health utility values with a crosssectional data set. Hence, the directional effects of insomnia on patients' utility over time, or, the responsiveness of the estimates could not be assessed in the

Table 4 Comparison of observed and predicted EQ-5D utility values

\begin{tabular}{|c|c|c|c|c|c|}
\hline & & Mean (SD) & Median & [Min-Max] & Range \\
\hline & Observed & $0.765(0.18)$ & 0.800 & {$[-0.040-1.000]$} & 1.040 \\
\hline \multirow[t]{4}{*}{ Estimation Sample } & Model I & $0.765(0.08)$ & 0.783 & [0.407-0.914] & 0.507 \\
\hline & Model II & $0.765(0.07)$ & 0.776 & [0.477-0.905] & 0.428 \\
\hline & Model III & $0.765(0.07)$ & 0.809 & {$[0.580-0.868]$} & 0.288 \\
\hline & Model IV & $0.771(0.07)$ & 0.772 & {$[0.324-0.895]$} & 0.571 \\
\hline \multirow[t]{4}{*}{ Validation Sample } & Model I & $0.766(0.08)$ & 0.786 & {$[0.367-0.891]$} & 0.524 \\
\hline & Model II & $0.765(0.07)$ & 0.776 & {$[0.488-0.896]$} & 0.408 \\
\hline & Model III & $0.764(0.07)$ & 0.811 & {$[0.571-0.858]$} & 0.287 \\
\hline & Model IV & $0.774(0.07)$ & 0.777 & {$[0.321-0.906]$} & 0.585 \\
\hline
\end{tabular}

For model specifications please refer to Table 1 
Table 5 Assessing model predictions

\begin{tabular}{|c|c|c|c|c|c|c|c|c|c|c|}
\hline & & \multirow[t]{2}{*}{$N$} & \multicolumn{2}{|c|}{ Model I } & \multicolumn{2}{|c|}{ Model II } & \multicolumn{2}{|c|}{ Model III } & \multicolumn{2}{|c|}{ Model IV } \\
\hline & & & MSE & MAE & MSE & MAE & MSE & MAE & MSE & MAE \\
\hline \multirow[t]{6}{*}{ Estimation Sample } & Full index & $1421(100 \%)$ & 0.024 & 0.109 & 0.025 & 0.110 & 0.026 & 0.112 & 0.024 & 0.110 \\
\hline & $<0.2$ & $10(0.68 \%)$ & $\mathrm{N} / \mathrm{A}$ & 0.545 & 0.375 & 0.566 & 0.424 & 0.567 & N/A & 0.526 \\
\hline & $0.2-0.4$ & $53(3.76 \%)$ & 0.184 & 0.350 & 0.146 & 0.366 & 0.155 & 0.377 & 0.192 & 0.392 \\
\hline & $0.4-0.6$ & $164(11.57 \%)$ & 0.070 & 0.232 & 0.066 & 0.235 & 0.068 & 0.241 & 0.072 & 0.250 \\
\hline & $0.6-0.8$ & $329(23.15 \%)$ & 0.008 & 0.066 & 0.007 & 0.063 & 0.007 & 0.064 & 0.005 & 0.057 \\
\hline & $0.8-1.0$ & 865 (60.85\%) & 0.013 & 0.083 & 0.013 & 0.084 & 0.014 & 0.085 & 0.013 & 0.087 \\
\hline \multirow[t]{6}{*}{ Validation Sample } & Full index & $1421(100 \%)$ & 0.025 & 0.110 & 0.024 & 0.110 & 0.025 & 0.111 & 0.023 & 0.108 \\
\hline & $<0.2$ & $10(0.73 \%)$ & N/A & 0.538 & 0.351 & 0.555 & 0.389 & 0.547 & N/A & 0.502 \\
\hline & $0.2-0.4$ & $53(3.70 \%)$ & 0.209 & 0.373 & 0.157 & 0.381 & 0.165 & 0.388 & 0.207 & 0.407 \\
\hline & $0.4-0.6$ & $163(11.44 \%)$ & 0.071 & 0.233 & 0.064 & 0.234 & 0.067 & 0.235 & 0.071 & 0.246 \\
\hline & $0.6-0.8$ & 326 (22.95\%) & 0.008 & 0.069 & 0.007 & 0.061 & 0.007 & 0.064 & 0.006 & 0.061 \\
\hline & $0.8-1.0$ & 869 (61.18\%) & 0.013 & 0.083 & 0.013 & 0.084 & 0.014 & 0.085 & 0.013 & 0.087 \\
\hline
\end{tabular}

-MSE = Mean square error

$-\mathrm{N} / \mathrm{A}=$ Estimates were not available due to the small sample size

$-\mathrm{MAE}=$ Mean absolute error

- Note: For model specifications please refer to Table 1

-Sample size for both the estimation and validation sample was randomly drawn (50\%) from the total qualified participants $(\mathrm{N}=2,842)$.

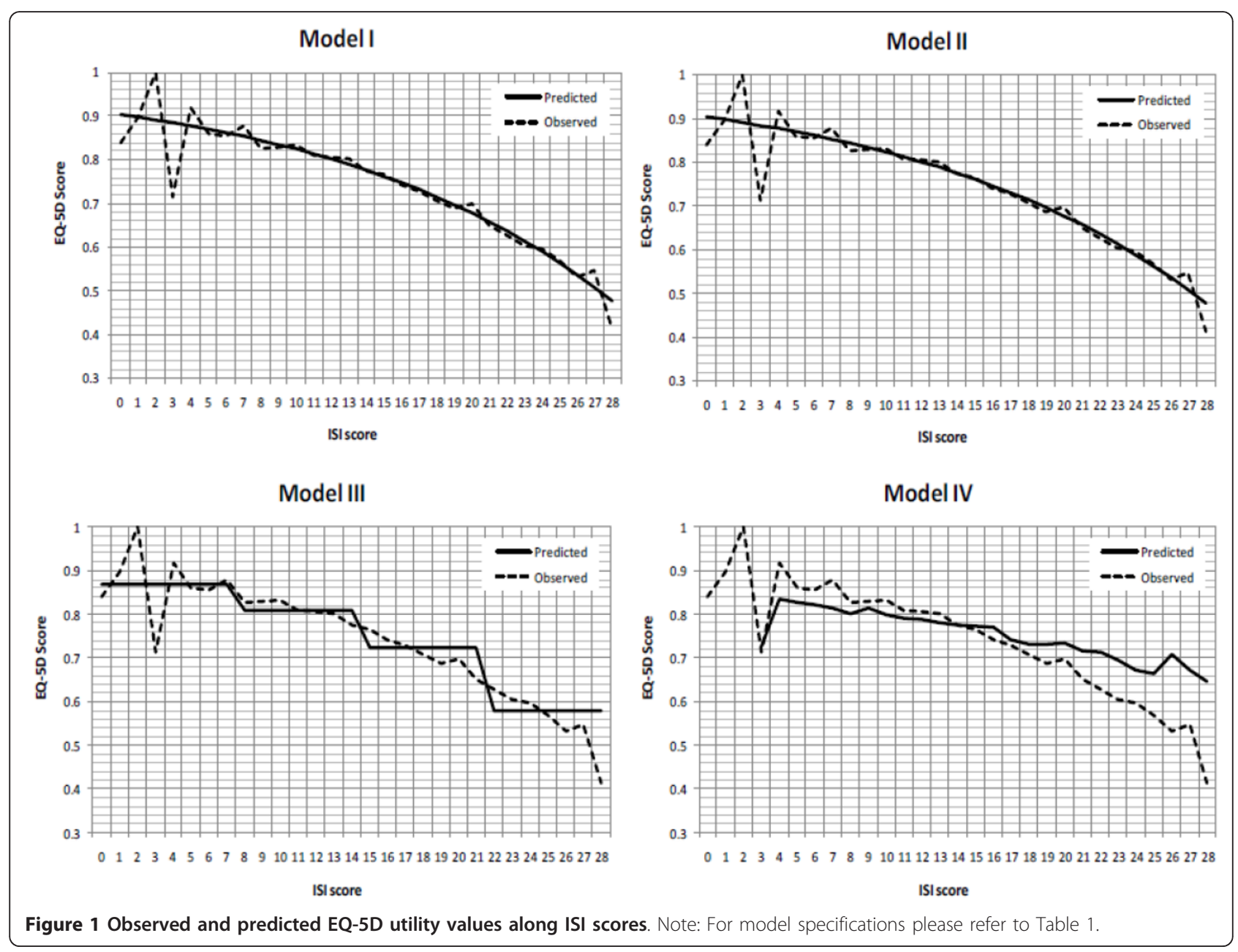




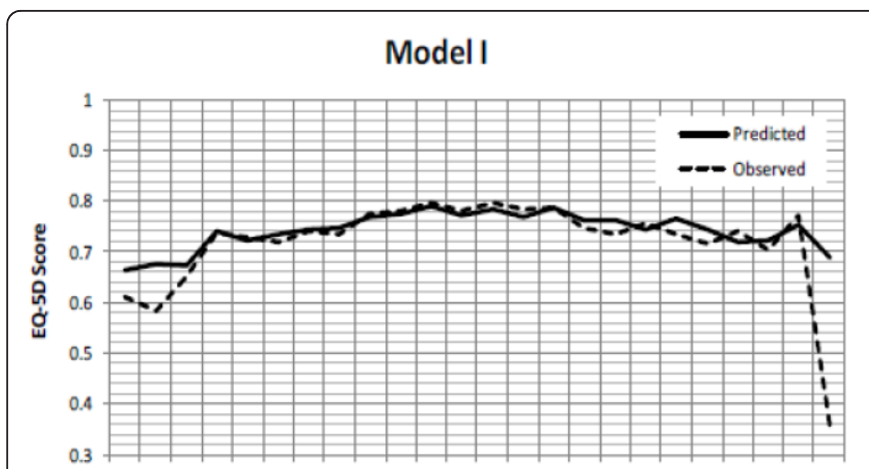

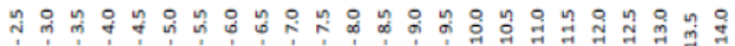

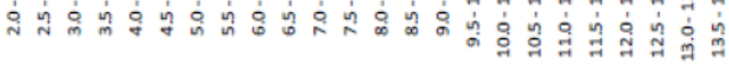

Duration (hours)

Model III

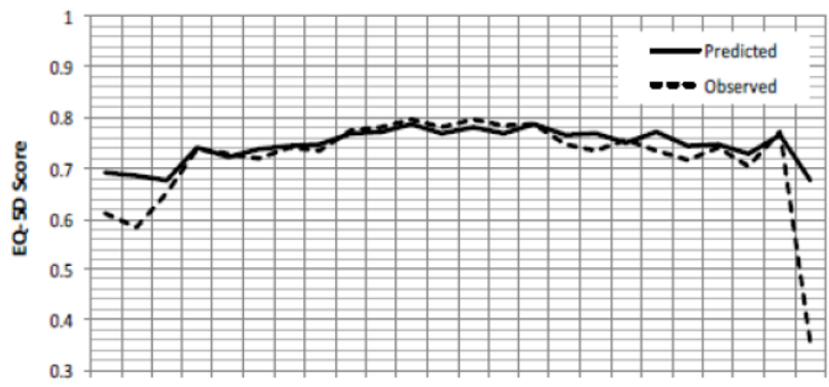

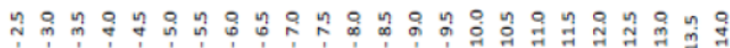

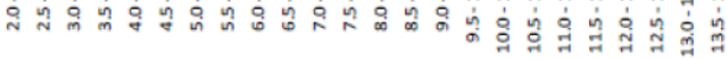

Duration (hours)

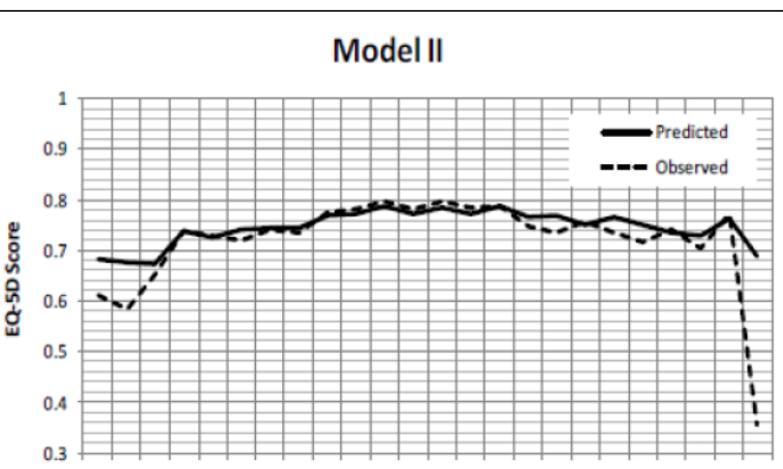

서수해

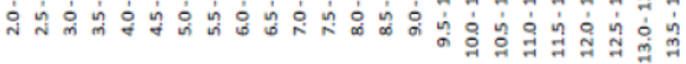

Duration (hours)

Model IV

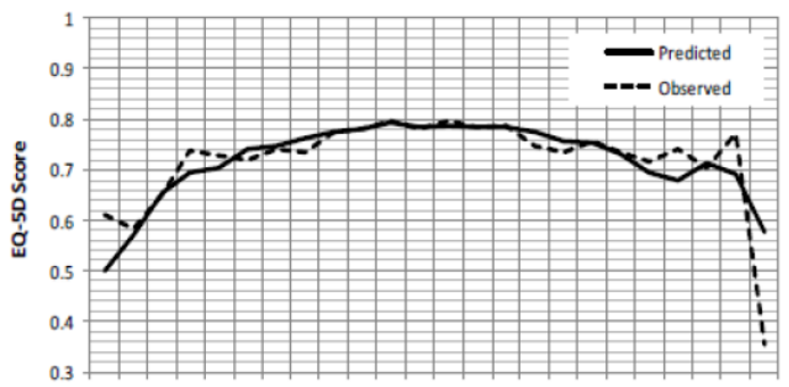

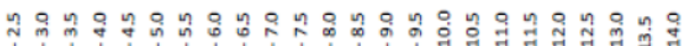

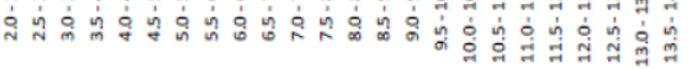

Duration (hours)

Figure 2 Observed and predicted EQ-5D utility values along sleep duration (in half-hour intervals). Note: For model specifications please refer to Table 1.

present study. It is therefore unclear whether the mathematical links reported in this study would vary over time.

Fifth, the current study relied on respondents' selfreported health status and not on physician assessment or medical records. Hence, the aim was not to identify causeand-effect relationships between sleep problems and other specific conditions or parameters. Interestingly, findings based on responses from such a convenient sample and not from a controlled clinical study were strikingly consistent with the study hypotheses, which provided vital validity of the analyses.

Sixth, treating the sleep quality variable in Model IV as continuous was a simplistic approach which assumed that each unit increase in sleep quality equally impacted the EQ-5D. Comparisons on the prediction results using the continuous and the categorical sleep quality showed that both approaches had equivalent predicted

Table 6 Sample utility computation

\begin{tabular}{|c|c|c|}
\hline Model & EQ-5D utility & Computation \\
\hline I & 0.772 & $\approx 1-\exp (-2.45614+0+0.14380-0.04075+0.13552+0.41963+0.24329+0.0766)$ \\
\hline$\|$ & 0.748 & $\approx 1-\exp \left(-2.34909+0.06077^{*} 16\right)$ \\
\hline III & 0.723 & $\approx 1-\exp (-2.0259+0.74122)$ \\
\hline IV & 0.690 & $\approx 1-\exp \left(-0.99338-0.00079 * 36-0.03204^{*} 1-0.22818^{*} 8.58+0.0133^{*} 8.58^{2}+0.42901 * 1-0.0314^{*} 1+0.00112^{*} 5+0.02498 * 8+0.05213^{*} 5\right)$ \\
\hline
\end{tabular}

Assume a 36-year old female had ISI scores of $0,3,1,4,3,3$, and 2 on the 7 items, respectively, resulting in an ISI summary score of 16 and a ISI class of 3, in combination with a report of 8.58 hours of sleep, $\geq 1$ comorbidity, 1 on overall sleep quality, 5 minutes of sleep onset latency during previous night, 8 on next day sleepiness, and 5 wake up times. 
outcomes; although, the trade-off was that distinctive effects on each sleep quality categories on the EQ-5D could not be separated under the continuous approach. Since the purpose of this study was to obtain the best estimates of the EQ-5D based on pre-defined predictors, we reported our findings under the continuous approach in Model IV.

Finally, the binary indicator of comorbidity used herein disregarded the possibility that a respondent with more than one comorbidity would likely report a worse utility, all other things being equal, than a respondent with one comorbidity. Likewise, this approach did not take into account which comorbidity was present, thus ignoring the possibility that some condition may have had a stronger impact on utility than others. It was beyond the scope of the present analysis to capture a more detailed quantification of these effects. Rather, an approximation of the EQ-5D utility values based on included parameters could be implemented using Model IV. Based on our findings, such approximation or estimation could be carried out in patients with either no comorbidities (i.e., primary insomnia) or in patients with one or more comorbidities (i.e., secondary insomnia). More importantly, the model was implementable regardless of whether the comorbidity conditions were listed in the current survey.

\section{Conclusion}

Estimating EQ-5D health state utility values using the algorithms presented herein permits comparisons of health outcomes in the absence of preference-based measures. Despite the aforementioned limitations, these algorithms give flexibility of computing EQ-5D health state utilities using different types of empirical insomnia data. Meanwhile the mapping relationship explored in this study serves as a "second-best" approach relative to direct elicitation of preference-based measures in clinical studies. Out-of-sample validation of these algorithms is encouraged to further establish the relationship between insomnia measures and the EQ-5D. This is especially true among patient groups with relatively lower observed health state utility values.

\footnotetext{
Author details

'Pharmerit North America, LLC,4350 East West Highway, Suite 430, Bethesda, MD 20814, USA. ${ }^{2}$ GlaxoSmithKline, Global Health Outcomes, Five Moore Drive, RTP, NC 27709, USA. ${ }^{3}$ Pharmerit Ltd., Tower House, Suite 8, Fishergate - York, YO10 4UA, UK. ${ }^{4}$ Department of Health Economics, HEDS, ScHARR, The University of Sheffield, Regent Court, 30 Regent Street, Sheffield S1 4DA, UK.
}

\section{Authors' contributions}

NYG, MFB, and BvH contributed to study design, analysis, interpretation, and manuscript writing. $\mathrm{XJ}$ and CFB contributed to study design, analysis, and interpretation. JAC contributed to analysis, interpretation, and manuscript writing. All authors have read and approved the final manuscript.

\section{Competing interests}

This manuscript was funded by GlaxoSmithKline. CFB is an employee of GlaxoSmithKline. NYG, MFB, XJ, JAC, and BVH are employees of Pharmerit which was paid a consulting fee by GlaxoSmithKline related to the development of this manuscript.

Received: 17 October 2011 Accepted: 30 December 2011

Published: 30 December 2011

\section{References}

1. American Psychiatric Association, American Psychiatric Association, Task Force on DSM-IV: Diagnostic and statistical manual of mental disorders DSMIV-TR. 4 edition. Washington, DC: American Psychiatric Association; 2000, text revision edn.

2. Moul DE, Hall M, Pilkonis PA, Buysse DJ: Self-report measures of insomnia in adults: rationales, choices, and needs. Sleep Med Rev 2004, 8:177-198.

3. Ancoli-Israel S, Roth T: Characteristics of insomnia in the United States: results of the 1991 National Sleep Foundation Survey. I. Sleep 1999, 22(Suppl 2):S347-S353.

4. Drake CL, Roehrs T, Roth T: Insomnia causes, consequences, and therapeutics: an overview. Depress Anxiety 2003, 18:163-176.

5. Roth T, Roehrs T: Insomnia: epidemiology, characteristics, and consequences. Clin Cornerstone 2003, 5:5-15.

6. Roth T: Insomnia: definition, prevalence, etiology, and consequences. $J$ Clin Sleep Med 2007, 3:S7-10.

7. Botteman MF, Ozminkowski RJ, Wang S, Pashos CL, Schaefer K, Foley DJ: Cost effectiveness of long-term treatment with eszopiclone for primary insomnia in adults: a decision analytical model. CNS Drugs 2007, 21:319-334.

8. Faubel R, Lopez-Garcia E, Guallar-Castillon P, Balboa-Castillo T, GutierrezFisac $J$, Banegas JR, et al: Sleep duration and health-related quality of life among older adults: a population-based cohort in Spain. Sleep 2009, 32:1059-1068.

9. Gibson GJ: Obstructive sleep apnoea syndrome: underestimated and undertreated. Br Med Bull 2004, 72:49-65.

10. Martin SA, Aikens JE, Chervin RD: Toward cost-effectiveness analysis in the diagnosis and treatment of insomnia. Sleep Med Rev 2004, 8:63-72.

11. Merlino G, Fratticci L, Lenchig C, Valente M, Cargnelutti D, Picello M, et al: Prevalence of 'poor sleep' among patients with multiple sclerosis: an independent predictor of mental and physical status. Sleep Med 2009, 10:26-34.

12. Silva GE, An MW, Goodwin JL, Shahar E, Redline S, Resnick H, et al: Longitudinal evaluation of sleep-disordered breathing and sleep symptoms with change in quality of life: the Sleep Heart Health Study (SHHS). Sleep 2009, 32:1049-1057.

13. Siriwardena AN, Apekey T, Tilling M, Harrison A, Dyas JV, Middleton HC, et al: Effectiveness and cost-effectiveness of an educational intervention for practice teams to deliver problem focused therapy for insomnia: rationale and design of a pilot cluster randomised trial. BMC Fam Pract 2009, 10:9.

14. Botteman M: Health economics of insomnia therapy: implications for policy. Sleep Med 2009, 10(Suppl 1):S22-S25.

15. Ware JE Jr, Sherbourne CD: The MOS 36-item short-form health survey (SF-36). I. Conceptual framework and item selection. Med Care 1992, 30:473-483.

16. Parrott AC, Hindmarch I: Factor analysis of a sleep evaluation questionnaire. Psychol Med 1978, 8:325-329.

17. Stewart AL, Ware JE: Measuring functioning and well-being the medical outcomes study approach Durham: Duke University Press; 1992.

18. Johns MW: A new method for measuring daytime sleepiness: the Epworth sleepiness scale. Sleep 1991, 14:540-545.

19. Miletin MS, Hanly PJ: Measurement properties of the Epworth sleepiness scale. Sleep Med 2003, 4:195-199.

20. Weaver DR: Reproductive safety of melatonin: a "wonder drug" to wonder about. J Biol Rhythms 1997, 12:682-689.

21. Buysse DJ, Reynolds CF III, Monk TH, Berman SR, Kupfer DJ: The Pittsburgh Sleep Quality Index: a new instrument for psychiatric practice and research. Psychiatry Res 1989, 28:193-213.

22. Bastien $\mathrm{CH}$, Vallieres A, Morin CM: Validation of the Insomnia Severity Index as an outcome measure for insomnia research. Sleep Med 2001, 2:297-307. 
23. Morin CM, Espie CA: Insomnia: A Clinician's Guide to Assessment and Treatment New York: Kluwer Academic/Plenum; 2003.

24. Sierra JC, Guillen-Serrano V, Santos-Iglesias P: [Insomnia Severity Index: some indicators about its reliability and validity on an older adults sample]. Rev Neurol 2008, 47:566-570.

25. Yu DS: Insomnia Severity Index: psychometric properties with Chinese community-dwelling older people. J Adv Nurs 2010, 66:2350-2359.

26. Neumann PJ, Goldie SJ, Weinstein MC: Preference-based measures in economic evaluation in health care. Annu Rev Public Health 2000, 21:587-611.

27. Brazier J: Measuring and valuing health benefits for economic evaluation Oxford: Oxford University Press; 2007.

28. Gold MR: Cost-effectiveness in health and medicine New York: Oxford University Press; 1996.

29. NICE: Guide to the Methods of Technology Appraisal. London, UK, National Institute for Clinical Excellence; 2004, Ref Type: Report.

30. Brooks R: EuroQol: the current state of play. Health Policy 1996, 37:53-72.

31. Dolan P: Modeling valuations for EuroQol health states. Med Care 1997, 35:1095-1108.

32. Kind P, Brooks R, Rabin R: EQ-5D concepts and methods a developmental history Dordrecht: Springer; 2005

33. Feeny D, Furlong W, Torrance GW, Goldsmith $\mathrm{CH}$, Zhu Z, DePauw S, et al: Multiattribute and single-attribute utility functions for the health utilities index mark 3 system. Med Care 2002, 40:113-128.

34. Shaw JW, Johnson JA, Coons SJ: US valuation of the EQ-5D health states: development and testing of the D1 valuation model. Med Care 2005, 43:203-220.

35. Kim SW, Shin IS, Kim JM, Kim YC, Kim KS, Kim KM, et al: Effectiveness of mirtazapine for nausea and insomnia in cancer patients with depression. Psychiatry Clin Neurosci 2008, 62:75-83.

36. Sullivan PW, Mulani PM, Fishman M, Sleep D: Quality of life findings from a multicenter, multinational, observational study of patients with metastatic hormone-refractory prostate cancer. Qual Life Res 2007 16:571-575.

37. McDaid C, Griffin S, Weatherly H, Duree K, van der BM, van HS, et al: Continuous positive airway pressure devices for the treatment of obstructive sleep apnoea-hypopnoea syndrome: a systematic review and economic analysis. Health Technol Assess 2009, 13:iii-xiv, 1.

38. Morgan K, Dixon S, Mathers N, Thompson J, Tomeny M: Psychological treatment for insomnia in the management of long-term hypnotic drug use: a pragmatic randomised controlled trial. Br J Gen Pract 2003, 53:923-928.

39. Morgan K, Dixon S, Mathers N, Thompson J, Tomeny M: Psychological treatment for insomnia in the regulation of long-term hypnotic drug use. Health Technol Assess 2004, 8:iii-68.

40. Snedecor SJ, Botteman MF, Bojke C, Schaefer K, Barry N, Pickard AS: Costeffectiveness of eszopiclone for the treatment of adults with primary chronic insomnia. Sleep 2009, 32:817-824.

41. Snedecor SJ, Botteman MF, Schaefer K, Sarocco P, Barry N, Pickard AS: Economic outcomes of eszopiclone treatment in insomnia and comorbid major depressive disorder. J Ment Health Policy Econ 2010, 13:27-35.

42. Deb P, Manning W, Norton E: Modeling health care costs and counts. ASHE 2006, Ref Type: Abstract.

43. McCullagh P, Nelder JA: Generalized linear models. 2 edition. London: Chapman and Hall; 1989.

44. Bamer AM, Johnson KL, Amtmann DA, Kraft GH: Beyond fatigue: Assessing variables associated with sleep problems and use of sleep medications in multiple sclerosis. Clin Epidemiol 2010, 2010:99-106.

45. Becker PM, Sattar M: Treatment of sleep dysfunction and psychiatric disorders. Curr Treat Options Neurol 2009, 11:349-357.

46. Benca RM, Peterson MJ: Insomnia and depression. Sleep Med 2008, 9(Suppl 1):S3-S9.

47. Brazier JE, Yang Y, Tsuchiya A, Rowen DL: A review of studies mapping (or cross walking) non-preference based measures of health to generic preference-based measures. Eur J Health Econ 2010, 11:215-225.

48. Mortimer D, Segal L: Comparing the incomparable? A systematic review of competing techniques for converting descriptive measures of health status into QALY-weights. Med Decis Making 2008, 28:66-89.
49. Katz DA, McHorney CA: The relationship between insomnia and healthrelated quality of life in patients with chronic illness. J Fam Pract 2002, 51:229-235.

50. Steptoe A, Peacey $V$, Wardle J: Sleep duration and health in young adults. Arch Intern Med 2006, 166:1689-1692.

51. Habte-Gabr E, Wallace RB, Colsher PL, Hulbert JR, White LR, Smith IM: Sleep patterns in rural elders: demographic, health, and psychobehavioral correlates. J Clin Epidemiol 1991, 44:5-13.

52. Segovia J, Bartlett RF, Edwards AC: The association between self-assessed health status and individual health practices. Can J Public Health 1989 80:32-37.

doi:10.1186/1477-7525-9-119

Cite this article as: Gu et al.: Mapping of the Insomnia Severity Index and other sleep measures to EuroQol EQ-5D health state utilities. Health and Quality of Life Outcomes 2011 9:119.

\section{Submit your next manuscript to BioMed Central and take full advantage of:}

- Convenient online submission

- Thorough peer review

- No space constraints or color figure charges

- Immediate publication on acceptance

- Inclusion in PubMed, CAS, Scopus and Google Scholar

- Research which is freely available for redistribution

Submit your manuscript at www.biomedcentral.com/submit
C) Biomed Central 